\title{
Plant-based diets, insulin sensitivity and inflammation in elderly men with chronic kidney disease
}

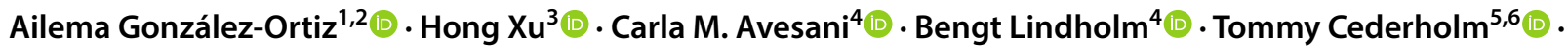

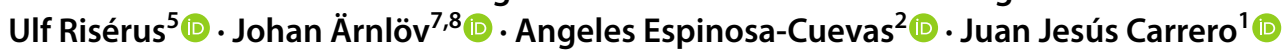

Received: 8 April 2020 / Accepted: 27 May 2020 / Published online: 8 June 2020

(C) The Author(s) 2020

\begin{abstract}
Background In persons with CKD, adherence to plant-based diets is associated with lower risk of CKD progression and death, but underlying mechanisms are poorly characterized. We here explore associations between adherence to plant-based diets and measures of insulin sensitivity and inflammation in men with CKD stages 3-5.

Methods Cross-sectional study including 418 men free from diabetes, aged 70-71 years and with cystatin-C estimated glomerular filtration rate (eGFR) $<60 \mathrm{~mL} / \mathrm{min} / 1.73 \mathrm{~m}^{2}$ and not receiving kidney-specific dietetic advice. Information from 7-day food records was used to evaluate the adherence to a plant-based diet index (PBDi), which scores positively the intake of plant-foods and negatively animal-foods. Insulin sensitivity and glucose disposal rate were assessed with the gold-standard hyperinsulinemic euglycemic glucose clamp technique. Inflammation was evaluated by serum concentrations of C-reactive protein (CRP) and interleukin (IL)-6. Associations were explored through linear regression and restricted cubic splines.

Results The majority of men had CKD stage 3a. Hypertension and cardiovascular disease were the most common comorbidities. The median PBDi was 38 (range 14-55). Across higher quintiles of PBDi (i.e. higher adherence), participants were less often smokers, consumed less alcohol, had lower BMI and higher eGFR ( $\mathrm{P}$ for trend $<0.05$ for all). Across higher PBDi quintiles, patients exhibited higher insulin sensitivity and lower inflammation ( $\mathrm{P}$ for trend $<0.05$ ). After adjustment for eGFR, lifestyle factors, BMI, comorbidities and energy intake, a higher PBDi score remained associated with higher glucose disposal rate and insulin sensitivity as well as with lower levels of IL-6 and CRP.
\end{abstract}

Juan Jesús Carrero

juan.jesus.carrero@ki.se

Ailema González-Ortiz

ailejgo@gmail.com

Hong Xu

hong.xu.2@ki.se

Carla M. Avesani

carla.avesani@ki.se

Bengt Lindholm

bengt.lindholm@ki.se

Tommy Cederholm

tommy.cederholm@pubcare.uu.se

Ulf Risérus

ulf.riserus@pubcare.uu.se

Johan Ärnlöv

johan.arnlov@ki.se

Angeles Espinosa-Cuevas

angeles.espinosac@incmnsz.mx
2 Nephrology and Mineral Metabolism Department, Instituto Nacional de Ciencias Médicas y Nutrición Salvador Zubirán, Mexico City, Mexico

3 Division of Clinical Geriatrics, Department of Neurobiology, Care Sciences and Society, Karolinska Institutet, Stockholm, Sweden

4 Renal Medicine and Baxter Novum, Clinical Science, Intervention and Technology, Karolinska Institutet, Stockholm, Sweden

5 Clinical Nutrition and Metabolism, Dept. of Public Health and Caring Sciences, Uppsala University, Uppsala, Sweden

6 Theme Ageing, Karolinska University Hospital, Stockholm, Sweden

7 School of Health and Social Studies, Dalarna University, Falun, Sweden

8 Division of Family Medicine and Primary Care, Department of Neurobiology, Care Sciences and Society (NVS), Karolinska Institutet, Alfred Nobels Allé 23, 14183 Huddinge, Sweden

1 Department of Medical Epidemiology and Biostatistics (MEB), Karolinska Institutet, Nobels väg 12A, Box 281,

17177 Stockholm, Sweden 
Conclusion In elderly men with non-dialysis CKD stages 3-5, adherence to a plant-based diet was associated with higher insulin sensitivity and lower inflammation, supporting a possible role of plant-based diets in the prevention of metabolic complications of CKD.

\title{
Graphic abstract
}

\section{Plant based diets, insulin sensitivity and inflammation in elderly men with chronic kidney disease.}

\author{
Methods \\ Design \\ Cross-sectional study \\ Study population \\ 418 men, with CKD 3-5, \\ free from diabetes, \\ aged $70-71$ years \\ Responding 7-day food records

\section{Outcomes} \\ Insulin sensitivity by the \\ hyperinsulinemic euglycemic \\ glucose clamp technique \\ Inflammation by C-reactive \\ protein and Interleukin-6

\section{Plant Based diet index}

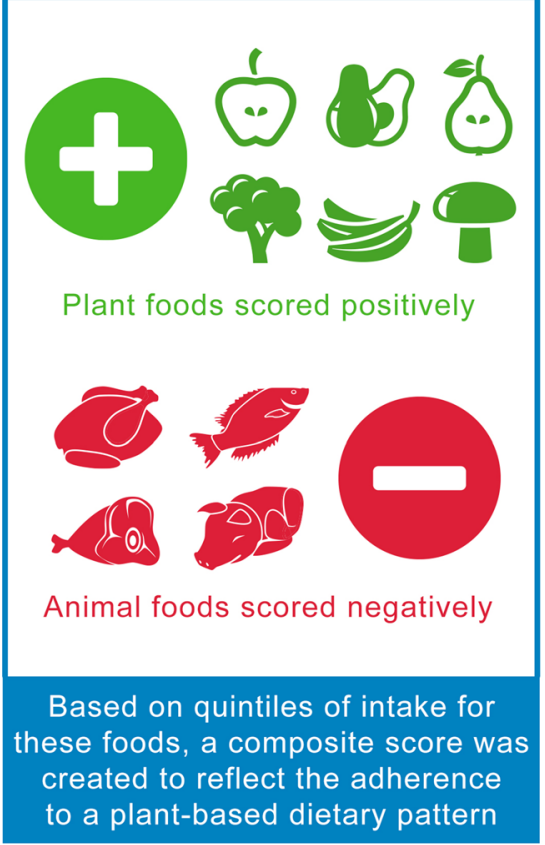 \\ Results and conclusion \\ In elderly men with non-dialysis CKD stages 3-5, adherence to a \\ plant-based diet was associated with higher insulin sensitivity and \\ weakly with lower inflammation, supporting a possible role of \\ plant-based diets in the prevention of metabolic complications of CKD.
}

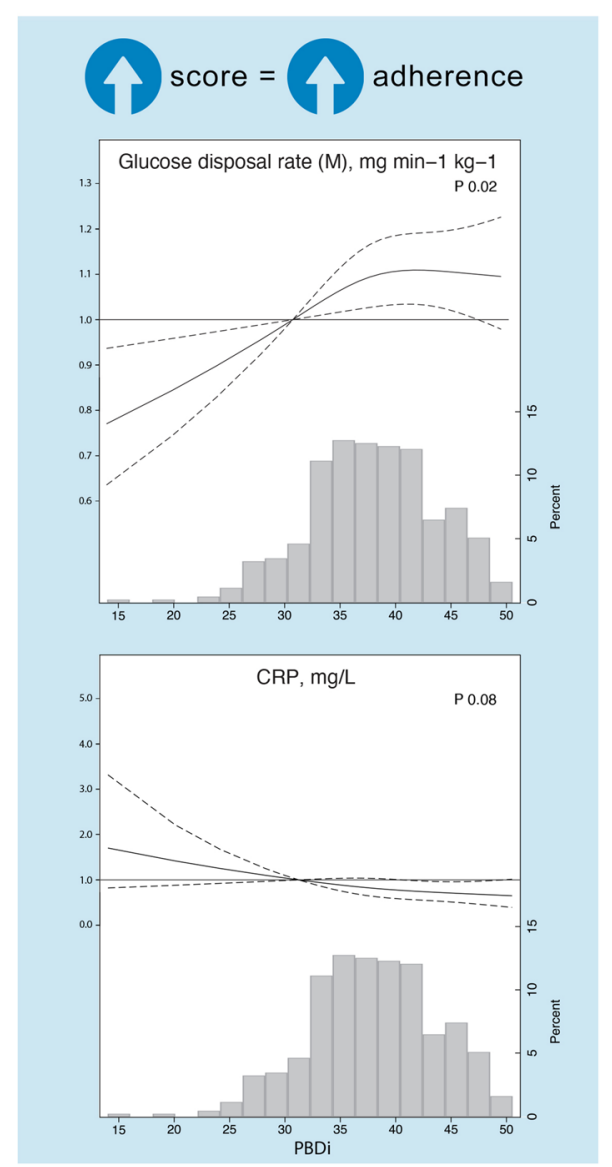

Keywords Fruit $\cdot$ Vegetable $\cdot$ Malnutrition $\cdot$ Protein-energy wasting $\cdot$ Potasssium $\cdot$ Restriction

\section{Introduction}

Dietary recommendations to treat chronic kidney disease (CKD) are focused on restricting dietary protein intake to avoid kidney overload and limiting the amount of nutrients that can accumulate into toxic levels (sodium, potassium, phosphorus) while ensuring sufficient energy intake [1, 2]. Without appropriate dietary counselling, such dietary restrictions can result in a diet of low food variety, often with a low intake of fruits and vegetables as a direct consequence of dietary potassium restriction [3, 4]. A number of recent observational studies suggest, however, that a more liberal consumption of plant-based foods in persons with CKD is associated with a lower risk of death and progression to end-stage kidney disease (ESKD) [5-7]. Mechanisms underlying these associations have, however, been poorly investigated.

One established mechanism by which increased intake of plant-foods may benefit CKD patients involves an overall reduction of dietary acid load [8], which in randomized trials lead to a better control of metabolic acidosis, reduction in urine excretion of angiotensinogen and estimated glomerular filtration rate (eGFR) preservation compared to usual care [9]. Plant-based diets have often 
been used as models for low-protein diets, which can delay the progression to ESKD and the need to start dialysis [10]. On the other hand, patients with CKD have progressively increased insulin resistance [11], and it has been proposed that a higher intake of plant foods may improve insulin sensitivity potentially through increasing the amount of short-chain fatty acids in plasma as a result of a higher fermentation of fiber by the microbiota $[12,13]$. While prospective studies in persons with diabetes suggest that dietary patterns rich in animal foods and poor in plants associate with insulin resistance [14], the studies in patients with CKD are scarce.. If any, a small $(n=39$ patients) 4-week trial [15] of fermentable fiber supplementation in persons with CKD G4 failed to observe changes in insulin resistance as estimated by the homeostatic model assessment (HOMA-IR). A third mechanisms by which plant-based diets may benefit persons with CKD is through the induction of lower systemic inflammation attributed to increased intake of fiber, phytochemicals, antioxidants and vitamins [16, 17]. Animal [18] and human studies in persons with CKD [19] show significant amelioration of oxidative stress and inflammation with fiber (amylose) supplementation. While dietary patterns that predict systemic inflammation have indeed been associated with the risk of CKD [20,21], as well as the speed of CKD progression [22], it is unknown whether plant-based diets may associate with lower inflammation in these patients.

The purpose of this study was to evaluate whether adherence to plant-based dietary patterns in persons with nondialysis CKD stages $3-5$ is associated with measures of insulin sensitivity and inflammation.

\section{Materials and methods}

\section{Study population}

This investigation was performed in the Uppsala Longitudinal Study of Adult Men (ULSAM) (http://www2. pubcare.uu.se/ULSAM/). The study, initiated in the 1970s, invited all 50-year-old men living in the Uppsala region to participate, and subsequent re-examinations were planned. The present analyses are based on the third examination cycle of the ULSAM cohort. During this examination, participants were 70-71 years of age (examinations performed during 1991-1995; $\mathrm{n}=1221$ ), and a detailed comorbid history, risk factor assessment and dietary records were collected simultaneously. For this specific analysis, we set some a priori exclusions: missing cystatin $\mathrm{C}$ to calculate GFR or having eGFR $>60 \mathrm{~mL} /$ $\mathrm{min} / 1.73 \mathrm{~m} \mathrm{[2]}$ as previously described [23] $(\mathrm{n}=594)$; missing 7-day dietary records or reporting extreme energy intake $<800$ or $>3800 \mathrm{kcal}(\mathrm{n}=121)$; having diabetes at the time of examination (defined as fasting plasma glucose $\geq 7.0 \mathrm{mmol} / \mathrm{L}, 2 \mathrm{~h}$ post-load glucose level $\geq 11.1 \mathrm{mmol} / \mathrm{L}$, or the use of oral hypoglycaemic agents or insulin; $n=88$ ). The present study therefore comprises 418 diabetes-free participants with CKD 3-5 and adequate dietary data reporting. None of the participants had a diagnosis of CKD or reported to have CKD, and thus we assume that they were not receiving any nephrology-specific dietetic advice. All participants gave written informed consent, and the Ethics Committee of Uppsala University approved the study protocols.

\section{Study exposure: adherence to a plant-based diet index}

All participants responded 7-day dietary records, using an optical readable form (OMR). The food record used was a pre-coded menu-book, prepared and previously used by the National Food Administration (NFA) [24]. Participants were given oral instructions by a dietitian on how to perform the dietary registration, and the amounts consumed were reported in household measurements or specified as portion sizes according. Nutrient consumption was standardized for total energy intake by regression analysis of the residual method [25]. Macronutrient intake as expressed as percent of energy, and consumption of potassium and fiber as grams per $1000 \mathrm{kcal}$. We also calculated average daily energy intake (DEI) $\mathrm{kcal} / \mathrm{kg} /$ day, daily protein intake (DPI) $\mathrm{g} / \mathrm{kg} / \mathrm{day}$.

Information from food records was used to compute the plant-based diet index (PBDi), as described previously [26]. Briefly, the PBDi is built by scoring the collective intake of plant and animal foods. The intake (in g/day) of 9 plant foods (cereal, fruit, vegetables, coffee and tea, refined grains, potato, juice, jam-sweet drinks and desserts, and chocolate-sweets-sugar) were transformed into quintiles of distribution. The sum of quintile values across these 9 food groups were scored positively (assigning a value of 1 for the first quintile, 2 for the second quintile, 3 for the third quintile, 4 for the fourth quintile and 5 for the fifth quintile). The intake (in g/day) of 5 animal food groups (meat, fish, egg, spreads, and dairy products-ice cream, cheese, milk) was transformed into quintiles of distribution. The sum of quintile values across these 5 food groups were scored reversely (assigning a value of 5 for the first quintile, 4 for the second quintile, 3 for the third quintile, 2 for the fourth quintile and 1 for the fifth quintile). The collective sum of these quintiles reflects the adherence to a plant-based dietary pattern with final scores ranging from 14 (lowest adherence) to 70 (highest adherence). 


\section{Study outcomes}

The first study outcome are measures of insulin sensitivity. The gold standard hyperinsulinemic euglycemic glucose clamp was performed as detailed by DeFronzo et al. [27] with slight modifications described elsewhere [28]. In brief, two intravenous infusion lines were placed, one into an antecubital vein and the other into a hand or wrist vein. After a 10-min priming infusion, insulin (Actrapid Human; Novo, Copenhagen, Denmark) infusion was held constant at a concentration of $660 \mathrm{pmol} / \mathrm{L}$ for $120 \mathrm{~min}$. Plasma glucose was measured every $5 \mathrm{~min}$ to be clamped at the euglycemic level $(5.1 \mathrm{mmol} / \mathrm{L})$ by infusion of variable amounts of $20 \%$ dextrose solution. The total body glucose disposal rate (M value), which is the basic clamp-derived IS index, was the average value of the glucose infusion rate during the final $60 \mathrm{~min}$ of the 120-min study (steady state). In addition, the Insulin Sensitivity Index (M/I ratio) was also calculated, which accounts for the insulin concentrations during the last $60 \mathrm{~min}$ of the clamp, and thus represents the amount of glucose metabolized (that is, taken up by the body) per unit of plasma insulin and is given in $\mathrm{mg} / \mathrm{kg}^{\prime}$ min per $\mathrm{mU} / \mathrm{L}$ of insulin multiplied by 100 . The clamp measurement in this cohort is considered to be reliable, as replication tests showed a high intra-individual variation of 0.93 and low coefficient of variation of 0.12 .

The second study outcome are measures of systemic inflammation. To this end, blood samples were drawn in the morning after overnight fasting. Plasma and serum determinations were performed at the Department of Clinical Chemistry, University Hospital, Uppsala, which is accredited according to the Swedish Board for Accreditation and Conformity Assessment (Swedac) standard ISO/IEC 17025. C-reactive protein (CRP) measurements were performed by latex enhanced reagent using a Behring BN ProSpec analyzer. The intra-assay coefficient of variation of the CRP method was $1.4 \%$ at both 1.23 and $5.49 \mathrm{mg} / \mathrm{L}$. Interleukina-6 (IL-6) was measured by an ELISA kit (IL-6 HS, R\&D Systems, Minneapolis, MN. The interassay coefficient of variation was $5 \%$.

\section{Study covariates}

Other study covariates were collected under standardized conditions, including anthropometric and biochemical measurements, and questionnaires regarding medical history, smoking habits, and physical activity level [29]. Body mass index (BMI) was calculated as body weight in kilograms divided by the square of height in meters. The waist and hip circumferences were measured in the supine position. The waist was measured midway between the lowest rib and the iliac crest and the hip over the widest part. The waist/hip ratio was calculated. The use of lipid-lowering and antihypertensive medications were collected with a medical questionnaire according to the, at that time, list of pharmaceutical specialties is available in Sweden (FASS 1992/1993). Insulin concentrations were determined using the Access Immunoassay System (Beckman-Coulter), which uses a chemiluminescent immunoenzymatic assay and Plasma glucose in samples from the oral glucose tolerance test was measured by the glucose dehydrogenase method (Gluc-DH, Merck, Darmstadt, Germany). Blood pressure measuring was attached to the subjects' non-dominant arm by a skilled lab technician. Systolic (SBP) and diastolic (DBP) blood pressures were measured every $30 \mathrm{~min}$ during daytime (06:00-23:00) and every hour during nighttime over $24 \mathrm{~h}$. Hyperlipidaemia was defined as serum cholesterol $>5.2 \mathrm{mmol} / \mathrm{L}$, triglycerides $>1.71 \mathrm{mmol} / \mathrm{L}$ or treatment with lipid-lowering medications. Smoking status was self-reported and defined as current smoking versus nonsmoking. Physical activity was self-reported and classified as sedentary and moderate vs regular and athletic [30]. Hypertension was defined as $24-\mathrm{h} \mathrm{SBP} \geq 130 \mathrm{mmHg}$, 24-h DBP $\geq 80 \mathrm{~mm} \mathrm{Hg}$, or use of antihypertensive medications [31]. CVD comorbid history was obtained by linkage to the Swedish Patient Register (ICD-9 codes 390-459, ICD-10 codes I00-I99).

\section{Statistical analysis}

Values are expressed as mean and standard deviation (SD) for continuous variables with normal distribution, median (interquartile range, IQR) for non-normal distribution variables and percentage of total for categorical. We report baseline characteristics of the sample according to quintiles $(\mathrm{Q})$ of plant based diet score and evaluate $\mathrm{P}$ for linear trends across these groups.

We used linear regression models to evaluate the association between PBDi (as a continuous variable, per score unit increase) and study outcomes. Because of skewed data distribution, eGFR, CRP, M and M/I were log-transformed before entering in the regression. Selection of covariates was done on the basis of biological consideration as confounders in the association of interest. Three stepwise models were investigated; In model 1, we considered multivariable adjustment for age and eGFR; In model 2, we further adjusted for total energy intake, lifestyle factors (physical activity, smoking and alcohol intake), BMI and comorbidities (CVD history and hypertension); in model 3, we further adjusted for the use of antihypertensive medication and lipid lowering agents.Finally, we investigated the relationship between PBDi and study outcomes graphically by the use of restricted cubic spline graphs with four degrees of freedom. Data are expressed as regression coefficients and 95\% confidence interval (95\% CIs). All statistical analysis 
were performed using STATA software (version 15.1; Stata Corp, College Station, TX).

\section{Results}

\section{Baseline characteristics}

After applying inclusion and exclusion criteria, the cohort consisted of 418 men free from diabetes, aged 70-71 years and with CKD stages $3-5$. The majority had CKD stage $3 a(n=322)$, followed by $3 b(n=86)$ and $4-5(n=10)$. The most common comorbidities were hypertension (77\%) and CVD (36\%).

Information from 7-day food records was used to compute the adherence to a PBDi. The PBDi ranged from 14 to 55, with a median of 38. Across increasing quintiles of PBDi (i.e. higher adherence, Table 1), participants showed a lower proportion of smokers, lower alcohol intake, lower BMI, waist circumference, and waist hip ratio, lower proportion of patients with hypertension, lower systolic blood pressure $(\mathrm{P}<0.05$ for all $)$ and a trend towards higher eGFR $(\mathrm{P}=0.06)$. No significant differences were found for physical activity, CVD history, glucose or insulinIn crude analysis, participants exhibited lower inflammation values (both CRP and IL-6) as well as higher both glucose disposal rate and insulin sensitivity $(\mathrm{P}<0.05$ for both, Table 1$)$. From a nutritional point of view, as expected, the intakes of fruit, vegetables, carbohydrates, potassium and fiber were greater with higher PBDi quintiles and the intake of fat and animal foods progressively lower. Notably no differences in protein intake were observed (Table 2).

Table 1 Baseline characteristics according to quintiles of plant-based diet index (PBDi) distribution

\begin{tabular}{|c|c|c|c|c|c|c|}
\hline Characteristic & Quintile $1(\mathrm{n}=78)$ & Quintile $2(\mathrm{n}=83)$ & Quintile $3(\mathrm{n}=82)$ & Quintile $4(n=91)$ & Quintile $5(\mathrm{n}=84)$ & $\mathrm{P}$ trend \\
\hline PBDi score & $31(14-33)$ & $35(34-36)$ & $38(37-39)$ & $41(40-43)$ & $46(44-55)$ & \\
\hline Age, years & $71 \pm 0.63$ & $71 \pm 0.63$ & $71 \pm 0.56$ & $71 \pm 0.52$ & $71 \pm 0.50$ & 0.19 \\
\hline BMI, $\mathrm{kg} / \mathrm{m}^{2}$ & $26.7 \pm 3.8$ & $26.2 \pm 3.8$ & $26.0 \pm 2.7$ & $26.2 \pm 3.0$ & $25.4 \pm 3.0$ & 0.05 \\
\hline Waist circumference, $\mathrm{cm}$ & $95.5(90-104)$ & $95(88-101)$ & $94(88-99)$ & 94 (89-99) & $93(87-98)$ & 0.02 \\
\hline Hip circumference, $\mathrm{cm}$ & $101(97-106)$ & $100(96-104)$ & $99(96-103)$ & $99(97-103)$ & $100(95-104)$ & 0.22 \\
\hline Waist/hip ratio & $0.96(0.91-0.99)$ & $0.96(0.9-0.98)$ & $0.93(0.9-0.96)$ & $0.95(0.91-0.98)$ & $0.93(0.9-0.96)$ & 0.03 \\
\hline Current smokers, n (\%) & $29(38)$ & $22(27)$ & $17(21)$ & $20(22)$ & $13(16)$ & $<0.01$ \\
\hline $\begin{array}{l}\text { Sedentary/moderate physical } \\
\text { activity, } \mathrm{n}(\%)\end{array}$ & $38(49)$ & $37(45)$ & $30(37)$ & $35(39)$ & $34(41)$ & 0.19 \\
\hline Alcohol intake, g/day & $6.3(1.9-11.1)$ & $4.6(1.4-11.9)$ & $4.4(1.0-8.2)$ & $4.4(1.0-8.0)$ & $2.7(0.6-6.9)$ & $<0.01$ \\
\hline Cardiovascular disease, $\%$ & $30(39)$ & $37(45)$ & $28(34)$ & $31(34)$ & $24(29)$ & 0.07 \\
\hline Hypertension, n (\%) & $65(83)$ & $66(80)$ & $63(77)$ & $69(76)$ & $58(69)$ & 0.03 \\
\hline ACEI/ARBs, n (\%) & $6(8)$ & $11(13)$ & $8(10)$ & $6(7)$ & $5(6)$ & 0.23 \\
\hline Beta blockers, n (\%) & $22(30)$ & $17(21)$ & $14(17)$ & $21(23)$ & $19(23)$ & 0.47 \\
\hline $\begin{array}{l}\text { Calcium channel antagonist, } \mathrm{n} \\
(\%)\end{array}$ & $13(18)$ & $10(12)$ & $5(6)$ & $14(15)$ & $10(12)$ & 0.55 \\
\hline Diuretics, n (\%) & $13(18)$ & $14(17)$ & $9(11)$ & $14(15)$ & $8(10)$ & 0.15 \\
\hline Lipid-lowering drugs, n (\%) & $6(8)$ & $8(10)$ & $7(9)$ & $14(15)$ & $7(8)$ & 0.56 \\
\hline \multicolumn{7}{|l|}{ Biochemical measurements } \\
\hline eGFR, $\mathrm{mL} / \mathrm{min}$ per $1.73 \mathrm{~m}^{2}$ & $50(44-55)$ & $51(45-57)$ & $51(46-56)$ & $51(47-57)$ & $54(49-57)$ & 0.06 \\
\hline Sistolic blood pressure & $140 \pm 17$ & $135 \pm 15$ & $135 \pm 15$ & $135 \pm 16$ & $132 \pm 15$ & 0.01 \\
\hline Diastolic blood pressure & $77 \pm 8$ & $76 \pm 9$ & $76 \pm 7$ & $76 \pm 8$ & $75 \pm 8$ & 0.07 \\
\hline Hyperlipidemia, n (\%) & $59(76)$ & $63(76)$ & $63(77)$ & $69(76)$ & $65(77)$ & 0.82 \\
\hline $\mathrm{CRP}, \mathrm{mg} / \mathrm{L}$ & $2.6(1.5-5.5)$ & $2.4(1.5-4.7)$ & $2.2(1.2-4.6)$ & $2.1(1.1-4.4)$ & $1.6(0.7-4.1)$ & $<0.01$ \\
\hline IL-6 ng/L & $4.5(2.5-9.2)$ & $4.1(2.4-7.4)$ & $3.7(2.2-5.9)$ & $3.4(2.1-5.2)$ & $3.7(2.1-7.4)$ & 0.03 \\
\hline Glucose, $\mathrm{mmol} / \mathrm{L}$ & $97.3 \pm 8.8$ & $95.0 \pm 9.9$ & $95.8 \pm 8.2$ & $95.0 \pm 9.5$ & $96.4 \pm 10.1$ & 0.53 \\
\hline Insulin, $\mu \mathrm{g} / \mathrm{L}$ & $43(29-62)$ & 45 (29-67) & $43(29-61)$ & $41(28-60)$ & $42(32-54)$ & 0.21 \\
\hline Glucose disposal rate & $4.9(3.6-5.9)$ & $5.3(4.1-6.8)$ & $5.5(4.4-6.6)$ & $5.2(3.9-7.0)$ & $5.6(4.2-6.7)$ & 0.03 \\
\hline $\begin{array}{l}\text { Insulin sensitivity, } \mathrm{mg} / \mathrm{min} / \mathrm{kg} \\
\mathrm{M} / \mathrm{I}, 100 \times \mathrm{mg} / \mathrm{kg} / \mathrm{min} \mathrm{mU} / \mathrm{L}\end{array}$ & $4.4(3.1-5.9)$ & $4.6(3.4-6.5)$ & $5.3(3.9-6.3)$ & $4.6(3.4-6.9)$ & $5.5(3.7-7.1)$ & 0.03 \\
\hline
\end{tabular}

Data are expressed as mean $\pm \mathrm{SD}$, median (25th, 75th centile), or number (\%), as appropriate

$B M I$ body mass index, ACE inhibitors angiotensin-converting-enzyme inhibitors, CRP C-reactive protein, Insulin sensitivity index (M/I) $100 \times \mathrm{mg} / \mathrm{kg} / \mathrm{min} \mathrm{mU} / \mathrm{L}$ 
Table 2 Nutrient intake characteristics according to quintiles of plant-based diet index (PBDi) distribution

\begin{tabular}{|c|c|c|c|c|c|c|}
\hline Characteristic & Quintile $1(\mathrm{n}=78)$ & Quintile $2(n=83)$ & Quintile $3(n=82)$ & Quintile $4(n=91)$ & Quintile $5(\mathrm{n}=84)$ & $\mathrm{P}$ trend \\
\hline PBDi score & $31(14-33)$ & $35(34-36)$ & $38(37-39)$ & $41(40-43)$ & $46(44-55)$ & \\
\hline Total energy intake, $\mathrm{kcal} / \mathrm{day}$ & $1690(1437-1860)$ & $1691(1333-1959)$ & $1621(1294-1876)$ & $1688(1395-1962)$ & $1806(1503-2056)$ & 0.06 \\
\hline $\begin{array}{l}\text { Dietary energy intake, } \mathrm{kcal} / \mathrm{kg} / \\
\text { day }\end{array}$ & $21(17-25)$ & $21(16-25)$ & $20(16-24)$ & $21(17-25)$ & $23.5(19-28)$ & 0.02 \\
\hline Dietary protein intake, $\mathrm{g} / \mathrm{kg} /$ day & $0.8(0.7-0.1)$ & $0.8(0.6-1)$ & $0.8(0.6-0.9)$ & $0.7(0.6-0.9)$ & $0.8(0.7-0.97)$ & 0.32 \\
\hline Total carbohydrates, $\%$ energy & $44.4(40.3-47.2)$ & $45.2(42.7-48.9)$ & $48.1(45.2-51.4)$ & $48.8(45.2-51.4)$ & $52.2(49.7-55.7)$ & $<0.01$ \\
\hline Total protein, $\%$ energy & $15.8(14.3-17.2)$ & $15.6(13.9-17.2)$ & $15.5(14.1-16.7)$ & $15.4(13.9-16.7)$ & $14.9(13.6-15.8)$ & $<0.01$ \\
\hline Fat, \% energy & $36.7(33.1-41.9)$ & $36.2(31.7-38.4)$ & $34.3(30.8-37.5)$ & $33.8(30.3-35.8)$ & $30.9(28.4-33.3)$ & $<0.01$ \\
\hline Saturated, $\%$ & $16(14-18)$ & $15(14-17)$ & $15(13-16)$ & $15(13-16)$ & $13(12-14)$ & $<0.01$ \\
\hline Monoinsaturared, $\%$ & $12(11-15)$ & $13(11-14)$ & $12(10-13)$ & $12(11.13)$ & $11(10.5-13)$ & $<0.01$ \\
\hline Polyunsaturated, $\%$ & $5(4-6)$ & $5(4-6)$ & $5(4-6)$ & $5(4-5)$ & $5(4-5)$ & $<0.01$ \\
\hline Potassium, mg/1000 kcal & $1496(1320-1692)$ & $1532(1350-1744)$ & $1559(1419-1722)$ & $1611(1476-1818)$ & $1640(1452-1839)$ & $<0.01$ \\
\hline Fiber intake, g/1000 kcal & $8.3(6.9-9.5)$ & $8.5(7.8-10.2)$ & $9.5(8.4-10.8)$ & $9.8(8.3-11.4)$ & $10.6(9.3-12.1)$ & $<0.01$ \\
\hline \multicolumn{7}{|l|}{ Foog groups g/1000 kcal } \\
\hline Meat & $63(47-79)$ & $57(43-79)$ & $49(39-65)$ & $49(40-68)$ & $46(36-56)$ & $<0.01$ \\
\hline Dairy products & $248(168-341)$ & $216(141-284)$ & $209(161-285)$ & $195(148-253)$ & $166(119-229)$ & $<0.01$ \\
\hline Egg & $13(8-26)$ & $11(7-17)$ & $7(4-19)$ & $10(5-14)$ & $6(4-11)$ & $<0.1$ \\
\hline Fruit & $48(17-75)$ & $48(27-76)$ & $71(30-112)$ & $82(40-109)$ & $86(45-128)$ & $<0.01$ \\
\hline Vegetable & $29(14-44)$ & $40(18-63)$ & $35(20-53)$ & $46(28-64)$ & $48(27-67)$ & $<0.01$ \\
\hline
\end{tabular}

Data are expressed as mean $\pm \mathrm{SD}$, median (25th, 75th centile), or number (\%), as appropriate

$B M I$ body mass index, ACE inhibitors angiotensin-converting-enzyme inhibitors, CRP C-reactive protein, Insulin sensitivity index (M/I) $100 \times \mathrm{mg} / \mathrm{kg}^{-1} / \mathrm{min}^{-1} \mathrm{mU} / \mathrm{L}$

\section{PBDi, insulin sensitivity and inflammation.}

We explored whether the association between PBDi score (as a continuous variable) and study outcomes was explained by confounders (Table 3). After adjustment for identified confounders in multivariable regression, every unit increase in the PBDi score remained associated with higher glucose disposal rate $[\beta(95 \% \mathrm{CI}) 0.007(0.001-0.01)]$ and insulin sensitivity index $[\beta(95 \% \mathrm{CI}) 0.008(0.001-0.02)]$. The fully adjusted model explained 42 and $39 \%$ of the variance of these outcomes, respectively. Accordingly, each unit increase in the PBDi was associated with lower levels of IL-6 $[\beta(95 \% \mathrm{CI})-0.17(-0.33$ to -0.001$)]$ and CRP $[\beta$ $(95 \% \mathrm{CI})-0.02(-0.04$ to -0.002$)]$. The shape of these associations are depicted graphically in Fig. 1; although impacted by broad confidence intervals, lower PBDi scores appear associated with worse insulin sensitivity and, more weakly, with increased measures of inflammation.

\section{Discussion}

In this observational study we show that among elderly men with non-dialysis CKD stages 3-5 free from diabetes, adherence to a PBDi was moderatedly associated with higher insulin sensitivity and lower systemic inflammation, suggesting a possible role of these diets in the prevention of metabolic complications in CKD.

A number of observational reports in the general population describe improved insulin sensitivity in persons consuming more plant-based foods [32-34], mostly attributed to the intake of cereals and fiber [35, 36]. A recent systematic review [37] reported consistency in the evidence relating vegetarianism with improved glycemic control and fasting glucose levels. In obese mice, the addition of soluble fiber to a high-fat diet significantly improved insulin sensitivity [38]. Our findings agree with this evidence and expand it to patients with CKD. Early small sample-size but welldesigned studies observed improved insulin sensitivity as assessed by the clamp technique among patients with nondialysis dependent CKD who followed a quasi-vegetarian very low protein diet supplemented with ketoanaloques [39, 40]. More recentry, a 4-week trial [15] of fermentable fiber supplementation in persons advanced CKD failed however to observe changes in insulin resistance. We argue that the small sample size $(n=39)$, the narrow range of eGFR included (15-25 $\mathrm{mL} / \mathrm{min})$, the short-term nature of the study and the surrogacy of the outcome (HOMA-IR) may have been responsible for this lack of association. Adherence to plant-based diets implies, by definition, a progressively lower intake of meat protein, which may have additional benefits for delaying ESKD [41]. In this sense, we note that diets 


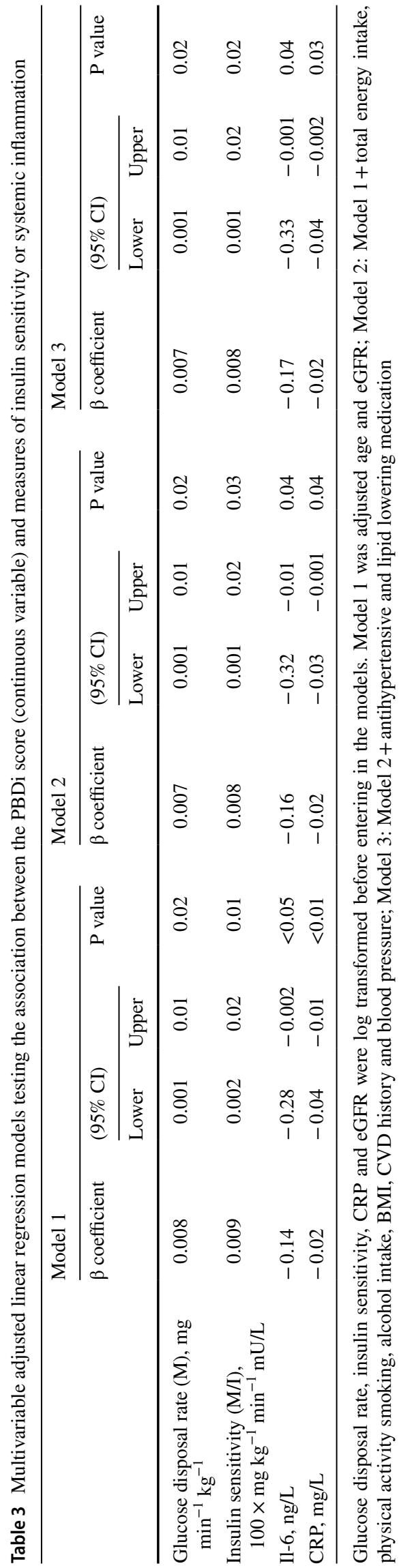

rich in animal foods have been shown to increase endogenous acid production with subsequent metabolic acidosis [42] and insulin resistance [43]. Evidence from a crossover trial of postmenopausal women suggests that replacing meat with soy results in improved insulin sensitivity [44]. In persons with CKD stages: 3-5, soy-based diets have been used as prototypes for low protein diets, documenting improved gut microbial profile $[45,46]$.

Single nutrients commonly rich in plant-based diets have been associated inversely with measures of systemic inflammation. Observational studies in persons with CKD $[16,47]$ reported that higher fiber intake was associated with lower both CRP and IL-6 levels. In a small interventional study, addition of fiber supplements (inulin) to a low protein diet resulted in reduced CRP levels in patients with CKD compared to a low protein diet alone [48]. Similar results were observed in animals [18] and in persons with CKD [19] supplemented with amylose. Our results of an association between the overall PBDi score and inflammation are novel, but agree with and expand this single-nutrient evidence. Mediterranean diets are also examples of a plantbased diet, as they promote a moderate consumption of animal food and a higher proportion of plant-foods. Landmark trials in the general population showed that Mediterranean diets can reduce markers of inflammation [49] and lower the risk of major cardiovascular events [50]. Evidence in CKD is scarce; in 40 persons CKD G2, a 90-day intervention of a Mediterranean diet [51] resulted in improvements in blood lipids and reductions in levels of CRP.

Strengths of our study include the stringent population selection (men of similar age), ascertainments of exposure from 7-day food records and of outcomes by gold-standard methods, particularly insulin sensitivity. Although we used a established plant-based diet scoring, there are other scores and dietary patterns in the literature [52] and this limits comparison with other studies. We acknowledge that our food composition software did not allow the separation of legumes and nuts, which may have reduced the sensitivity of our scoring, nor give distinct consideration of lesshealthy plant foods such as potatoes (especially consumed as French fries) and added sugars. Associations are crosssectional and sex- and cohort-specific in a population of men of Nordic upbringing and free from diabetes. Extrapolation to women, other diseases, regions and periods should be done with caution. A priori, we do not anticipate associations to differ between sexes. However, aging encompasses changes in inflammation markers, physical activity and/or frailty. Studies evaluating these associations in women as well as in younger populations should follow. Persons with a higher PBDi score also had other typical features of healthy lifestyle, such as less smoking, being more physically active, consuming less alcohol or having a lower BMI. A higher PBDi score may also reflect socioeconomic differences. 

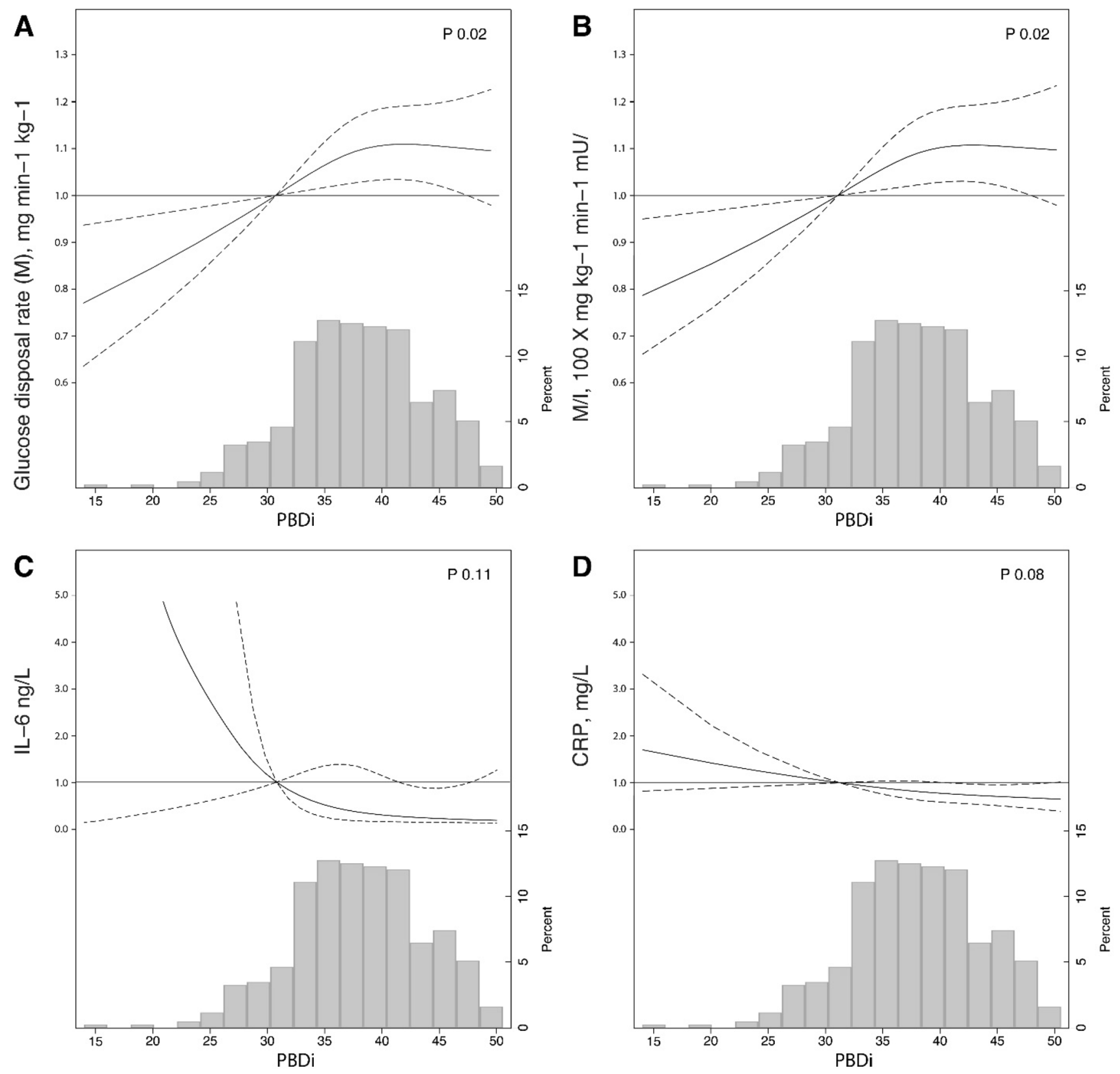

Fig. 1 Restricted cubic spline curves showing adjusted $\beta$ coefficient (bold line) and 95\% confidence intervals (dashed lines) for glucose disposal (a), insulin sensitivity indes (M/I, b), Il-6 (c) and CRP (d) associated with a plant-based diet index (PBDi) score. Covariates

include age, eGFR, total energy intake, physical activity, smoking status, alcohol intake, BMI, CVD history, blood pressure, antihypertensives and lipid lowering medication

Although we tried to adjust for some of these factors in our multivariable analyses, a limitation of this and any observational study is the presence of unknown confounding our inability to establish causation. The results may to some extent reflect a more conscious attitude to lifestyle risks in general among those with a lower PBDi score. We recognize that the risk magnitude of our associations, although statistically significant, is not big and (as evidenced by the spline curves) is affected by broad confidence intervals. This can be

attributed to a relatively low sample size in our cohort, but also perhaps to the fact that diet is one of many and probably not the most important factor leading to insulin resistance and inflammation in such polymorbid and complex patients.

To date, only one controlled study in patients with macroalbuminuria and CKD G3 has explored the effect of increasing the intake of fruits and vegetables. Randomization for three years to usual care or interventions designed to reduce dietary acid by $50 \%$, using either sodium bicarbonate 
or alkali-rich fruits and vegetables [9] resulted in similar control of metabolic acidosis and eGFR preservation and to a greater extent than usual care. Of note, a 5-year extension of the follow up of this trial [53] not only confirmed these findings, but also showed that the group receiving fruits and vegetables had better systolic blood pressure and body mass index control, improved lipid profile and higher serum levels of vitamin $\mathrm{K}_{1}$ than those under bicarbonate therapy or usual care [53].

To conclude, adherence to a plant-based diet, as represented through the PBDi score, was associated with higher insulin sensitivity and lower systemic inflammation in elderly men with CKD stages 3-5. Such findings suggest a possible role of these diets in the prevention of metabolic complications for these patients, and adds to recent observational studies associating plant-based diets with a lower risk of death and progression to ESKD. Whether the associations are causal can only be answered in an adequately designed and powered interventional study.

Acknowledgements This work was supported by a grant from the Swedish Research Council (2019-01059). AGO was supported by The National Council of Science and Technology (CONACYT), CVU 373297. School of Medicine. Programa de Maestría y Doctorado en Ciencias Médicas, Odontológicas y de la Salud. Baxter Novum is the result of a grant from Baxter Healthcare to Karolinska Institutet.

Author contributions AGO and HX: Participated in study conception and design the research generation, analysis of the data and writing the paper. JJC Participated in study conception and design, revision and analysis of the data, writing the paper and approval of the final version of the manuscript. CMA,BL,TC,UR,JA and AEC provided data, participated in interpretation of the data and/or critical revision of the manuscript to its final form. All authors read and approved the final manuscript.

Funding Open access funding provided by Karolinska Institute.

\section{Compliance with ethical standards}

Conflict of interest J.J.C reports grant funding from AstraZeneca, ViforPharma and Astellas, consulting for Baxter and AstraZeneca, and speaker fees for Abbott, Nutricia, AstraZeneca and ViforPharma. all outside the submitted work. BL is affiliated with Baxter Healthcare Corporation. AEC acknowledges speaker honoraria from Abbott Laboratories and AbbVie. None of the other authors declare any conflict of interest.

Open Access This article is licensed under a Creative Commons Attribution 4.0 International License, which permits use, sharing, adaptation, distribution and reproduction in any medium or format, as long as you give appropriate credit to the original author(s) and the source, provide a link to the Creative Commons licence, and indicate if changes were made. The images or other third party material in this article are included in the article's Creative Commons licence, unless indicated otherwise in a credit line to the material. If material is not included in the article's Creative Commons licence and your intended use is not permitted by statutory regulation or exceeds the permitted use, you will need to obtain permission directly from the copyright holder. To view a copy of this licence, visit http://creativecommons.org/licenses/by/4.0/.

\section{References}

1. Kopple JD (2001) National kidney foundation K/DOQI clinical practice guidelines for nutrition in chronic renal failure. Am J Kidney Dis 37:S66-70. https://doi.org/10.1053/ajkd.2001.20748

2. Cupisti A et al (2018) Nutritional treatment of advanced CKD: twenty consensus statements. J Nephrol 31:457-473. https://doi. org/10.1007/s40620-018-0497-z

3. Anderson CAM, Nguyen HA (2018) Nutrition education in the care of patients with chronic kidney disease and end-stage renal disease. Semin Dial 31:115-121. https://doi.org/10.1111/ sdi. 12681

4. Fernandes AS, Ramos CI, Nerbass FB, Cuppari L (2018) Diet quality of chronic kidney disease patients and the impact of nutritional counseling. J Ren Nutr 28:403-410. https://doi. org/10.1053/j.jrn.2017.10.005

5. Kelly JT et al (2017) Healthy dietary patterns and risk of mortality and ESRD in CKD: a meta-analysis of cohort studies. Clin J Am Soc Nephrol 12:272-279. https://doi.org/10.2215/CJN.06190616

6. Yuzbashian E, Asghari G, Mirmiran P, Amouzegar-Bahambari P, Azizi F (2018) Adherence to low-sodium dietary approaches to stop hypertension-style diet may decrease the risk of incident chronic kidney disease among high-risk patients: a secondary prevention in prospective cohort study. Nephrol Dial Transplant 33:1159-1168. https://doi.org/10.1093/ndt/gfx352

7. Tyson CC et al (2019) DASH diet and blood pressure among black Americans with and without chronic kidney disease: the Jackson Heart Study. Am J Hypertens. https://doi.org/10.1093/ajh/hpz090

8. Wesson DE, Simoni J (2010) Acid retention during kidney failure induces endothelin and aldosterone production which lead to progressive GFR decline, a situation ameliorated by alkali diet. Kidney Int 78:1128-1135. https://doi.org/10.1038/ki.2010.348

9. Goraya N, Simoni J, Jo CH, Wesson DE (2014) Treatment of metabolic acidosis in patients with stage 3 chronic kidney disease with fruits and vegetables or oral bicarbonate reduces urine angiotensinogen and preserves glomerular filtration rate. Kidney Int 86:1031-1038. https://doi.org/10.1038/ki.2014.83

10. Hahn D, Hodson EM, Fouque D (2018) Low protein diets for nondiabetic adults with chronic kidney disease. Cochrane Database Syst Rev 10:Cd001892. https://doi.org/10.1002/14651858.CD001 892.pub4

11. DeFronzo RA et al (1981) Insulin resistance in uremia. J Clin Invest 67:563-568. https://doi.org/10.1172/JCI110067

12. Lau $\mathrm{C}$ et al (2005) Dietary glycemic index, glycemic load, fiber, simple sugars, and insulin resistance: the Inter99 study. Diabetes Care 28:1397-1403. https://doi.org/10.2337/diacare.28.6.1397

13. Morrison DJ, Preston T (2016) Formation of short chain fatty acids by the gut microbiota and their impact on human metabolism. Gut Microbes 7:189-200. https://doi.org/10.1080/19490 976.2015.1134082

14. Adeva-Andany MM et al (2019) Effect of diet composition on insulin sensitivity in humans. Clin Nutr ESPEN 33:29-38. https ://doi.org/10.1016/j.clnesp.2019.05.014

15. Poesen $\mathrm{R}$ et al (2016) The influence of prebiotic arabinoxylan oligosaccharides on microbiota derived uremic retention solutes in patients with chronic kidney disease: a randomized controlled trial. PLoS ONE 11:e0153893. https://doi.org/10.1371/journ al.pone.0153893 
16. $\mathrm{Xu} \mathrm{H}$ et al (2014) Dietary fiber, kidney function, inflammation, and mortality risk. Clin J Am Soc Nephrol 9:2104-2110. https:// doi.org/10.2215/CJN.02260314

17. Lattimer JM, Haub MD (2010) Effects of dietary fiber and its components on metabolic health. Nutrients 2:1266-1289. https ://doi.org/10.3390/nu2121266

18. Vaziri ND et al (2014) High amylose resistant starch diet ameliorates oxidative stress, inflammation, and progression of chronic kidney disease. PLoS ONE 9:e114881. https://doi.org/10.1371/ journal.pone. 0114881

19. Tayebi Khosroshahi $\mathrm{H}$ et al (2018) Effect of high amylose resistant starch (HAM-RS2) supplementation on biomarkers of inflammation and oxidative stress in hemodialysis patients: a randomized clinical trial. Hemodial Int 22:492-500. https://doi.org/10.1111/ hdi. 12653

20. $\mathrm{Xu} \mathrm{H}$ et al (2015) A proinflammatory diet is associated with systemic inflammation and reduced kidney function in elderly adults. J Nutr 145:729-735. https://doi.org/10.3945/jn.114.205187

21. Mazidi M, Shivappa N, Wirth MD, Hebert JR, Kengne AP (2018) Greater dietary inflammatory index score is associated with higher likelihood of chronic kidney disease. Br J Nutr 120:204-209. https ://doi.org/10.1017/s0007114518001071

22. Rouhani MH et al (2019) Dietary inflammatory index and its association with renal function and progression of chronic kidney disease. Clin Nutr ESPEN 29:237-241. https://doi.org/10.1016/j. clnesp.2018.09.001

23. Larsson, Grubb, Hansson (2004) Calculation of glomerular filtration rate expressed in $\mathrm{mL} / \mathrm{min}$ from plasma cystatin $\mathrm{C}$ values in $\mathrm{mg} / \mathrm{L}$. Scand J Clin Lab Invest 64:25-30. https://doi. org/10.1080/00365510410003723

24. Nydahl M, Gustafsson IB, Mohsen R, Becker W (2009) Comparison between optical readable and open-ended weighed food records. Food Nutr Res. https://doi.org/10.3402/fnr.v53i0.1889

25. Willet W, Howe GR, Laurence HK (1228s) Adjustment for total energy intake in epidemiologic studies. Am J Clin Nutr $65: 1220$ s-1228s

26. Kim $\mathrm{H}$ et al (2019) Plant-based diets and incident CKD and kidney function. Clin J Am Soc Nephrol 14:682-691. https://doi. org/10.2215/CJN.12391018

27. DeFronzo RA, Tobin JD, Andres R (1979) Glucose clamp technique: a method for quantifying insulin secretion and resistance. Am J Physiol 237:E214-223. https://doi.org/10.1152/ajpen do.1979.237.3.E214

28. Jia T et al (2014) Validation of insulin sensitivity surrogate indices and prediction of clinical outcomes in individuals with and without impaired renal function. Kidney Int 86:383-391. https:// doi.org/10.1038/ki.2014.1

29. Vessby B, Tengblad S, Lithell H (1994) Insulin sensitivity is related to the fatty acid composition of serum lipids and skeletal muscle phospholipids in 70-year-old men. Diabetologia 37:1044-1050

30. Byberg L, Zethelius B, McKeigue PM, Lithell HO (2001) Changes in physical activity are associated with changes in metabolic cardiovascular risk factors. Diabetologia 44:2134-2139

31. Whelton PK et al (2018) 2017 ACC/AHA/AAPA/ABC/ACPM/ AGS/APhA/ASH/ASPC/NMA/PCNA guideline for the prevention, detection, evaluation, and management of high blood pressure in adults. J Am Coll Cardiol 71:e127-e248. https://doi. org/10.1016/j.jacc.2017.11.006

32. Satija A et al (2016) Plant-based dietary patterns and incidence of type 2 diabetes in US men and women: results from three prospective cohort studies. PLoS Med 13:e1002039. https://doi. org/10.1371/journal.pmed.1002039

33. Barnard ND, Scialli AR, Turner-McGrievy G, Lanou AJ, Glass J (2005) The effects of a low-fat, plant-based dietary intervention on body weight, metabolism, and insulin sensitivity. Am J Med 118:991-997. https://doi.org/10.1016/j.amjmed.2005.03.039

34. Chen $Z$ et al (2018) Plant versus animal based diets and insulin resistance, prediabetes and type 2 diabetes: the Rotterdam Study. Eur J Epidemiol 33:883-893. https://doi.org/10.1007/s1065 4-018-0414-8

35. Broekaert WF et al (2011) Prebiotic and other health-related effects of cereal-derived arabinoxylans, arabinoxylan-oligosaccharides, and xylooligosaccharides. Crit Rev Food Sci Nutr 51:178-194. https://doi.org/10.1080/10408390903044768

36. Kim YA, Keogh JB, Clifton PM (2018) Probiotics, prebiotics, synbiotics and insulin sensitivity. Nutr Res Rev 31:35-51. https ://doi.org/10.1017/s095442241700018x

37. Viguiliouk E et al (2019) Effect of vegetarian dietary patterns on cardiometabolic risk factors in diabetes: a systematic review and meta-analysis of randomized controlled trials. Clin Nutr 38:11331145. https://doi.org/10.1016/j.clnu.2018.05.032

38. Xu C et al (2020) Combined soluble fiber-mediated intestinal microbiota improve insulin sensitivity of obese mice. Nutrients. https://doi.org/10.3390/nu12020351

39. Gin $\mathrm{H}$ et al (1987) Low protein and low phosphorus diet in patients with chronic renal failure: influence on glucose tolerance and tissue insulin sensitivity. Metabolism 36:1080-1085. https:// doi.org/10.1016/0026-0495(87)90029-1

40. Rigalleau V et al (1997) A low-protein diet improves insulin sensitivity of endogenous glucose production in predialytic uremic patients. Am J Clin Nutr 65:1512-1516. https://doi.org/10.1093/ ajen/65.5.1512

41. Lew QJ et al (2017) Red meat intake and risk of ESRD. J Am Soc Nephrol 28:304-312. https://doi.org/10.1681/asn.2016030248

42. Adeva MM, Souto G (2011) Diet-induced metabolic acidosis. Clin Nutr 30:416-421. https://doi.org/10.1016/j.clnu.2011.03.008

43. Akter $\mathrm{S}$ et al (2016) High dietary acid load is associated with insulin resistance: the Furukawa Nutrition and Health Study. Clin Nutr 35:453-459. https://doi.org/10.1016/j.clnu.2015.03.008

44. van Nielen M, Feskens EJ, Rietman A, Siebelink E, Mensink M (2014) Partly replacing meat protein with soy protein alters insulin resistance and blood lipids in postmenopausal women with abdominal obesity. J Nutr 144:1423-1429. https://doi. org/10.3945/jn.114.193706

45. Marzocco S et al (2013) Very low protein diet reduces indoxyl sulfate levels in chronic kidney disease. Blood Purif 35:196-201. https://doi.org/10.1159/000346628

46. Black AP et al (2018) Does low-protein diet influence the uremic toxin serum levels from the gut microbiota in nondialysis chronic kidney disease patients? J Ren Nutr 28:208-214. https:// doi.org/10.1053/j.jrn.2017.11.007

47. Krishnamurthy VM et al (2012) High dietary fiber intake is associated with decreased inflammation and all-cause mortality in patients with chronic kidney disease. Kidney Int 81:300-306. https://doi.org/10.1038/ki.2011.355

48. Lai $\mathrm{S}$ et al (2019) Effect of low-protein diet and inulin on microbiota and clinical parameters in patients with chronic kidney disease. Nutrients. https://doi.org/10.3390/nu11123006

49. Fuentes GC et al (2020) Prospective association of physical activity and inflammatory biomarkers in older adults from the PREDIMED-Plus study with overweight or obesity and metabolic syndrome. Clin Nutr. https://doi.org/10.1016/j.clnu.2020.01.015

50. Appel LJ et al (1997) A clinical trial of the effects of dietary patterns on blood pressure. DASH Collaborative Research Group. N Eng J Med 336:1117-1124. https://doi.org/10.1056/nejm199704 173361601

51. Mekki K, Bouzidi-bekada N, Kaddous A, Bouchenak M (2010) Mediterranean diet improves dyslipidemia and biomarkers in chronic renal failure patients. Food Funct 1:110-115. https://doi. org/10.1039/c0fo00032a 
52. Williams KA Sr, Patel H (2017) Healthy plant-based diet: what does it really mean? J Am Coll Cardiol 70:423-425. https://doi. org/10.1016/j.jacc.2017.06.006

53. Goraya N, Munoz-Maldonado Y, Simoni J, Wesson DE (2019) Fruit and vegetable treatment of chronic kidney disease-related metabolic acidosis reduces cardiovascular risk better than sodium bicarbonate. Am J Nephrol 49:438-448. https://doi. org/10.1159/000500042
Publisher's Note Springer Nature remains neutral with regard to jurisdictional claims in published maps and institutional affiliations. 